$\xi=-1$

\title{
Low Complexity Signal Detector for MIMO MCCDMA System for Longer Delay Channel
}

\author{
Papa Rao Challagundla ${ }^{*}$, P.Sumithabhashini ${ }^{2}$, P.Chandrasekhar Reddy ${ }^{3}$ \\ ${ }^{I}$ Department of ECE, Research Scholar in JNTUH, Hyderabad, India \\ ${ }^{2}$ Department of ECE, ACE Engineering College, Hyderabad, India \\ ${ }^{3}$ Department of ECE, JNTUHCE, Hyderabad, India \\ *Corresponding Author E-mail: ${ }^{1}$ paparao.challagundla@gmail.com
}

\begin{abstract}
In recent years Multiple-input multiple-output (MIMO) Multi carrier code division multiple access (MCCDMA), which combines the advantage of diversities with high spectral efficiency has drawn great attention. In this paper we introduce a novel low-complexity multiple-input multiple-output (MIMO) MMSE detector tailored for MCDCDMA systems, suitable for frequency selective channel. The proposed detector begins with estimation of the minimum mean square error (MMSE) on less reliable symbols followed by iterative decorrelation as post-detection processing for mitigating multiple access interferences. Efficient high-throughput VLSI architecture is used to achieve superior performance compared to the conventional MMSE detectors. The performance of the proposed MMSE detector is close to the efficient maximum likelihood, with significant complexity reduction over higher order constellations. The efficiency of MIMO MCCDMA over high order constellations and its quality retentions are verified through MATLAB BER simulation.
\end{abstract}

Keywords: Multiple-input multiple-output (MIMO), minimum mean square error (MMSE), and Code Division Multiple Accesses, Spreading gain Maximum Likelihood, Multiple Access Interference (MAI) etc.

\section{Introduction}

Recent trends emerged in wireless communication system requires high throughput transmission rate over high mobility channels. Orthogonal frequency division multiplexing (OFDM) is the most widely used predominant technique used in many existing broadband transmission methodologies due to its robustness to the frequency selective fading; inter symbol interference (ISI) etc. In multi-carrier code division multiple access (MCCDMA), spreading gain is introduced in both frequency and time domain in addition with the OFDM modulation for getting high speed data rate and multi subscriber accessing scheme.

In high data rate and multi user environment, the transmitted symbols often experience both selective fading and channel frequency offsets due to multipath effects and Doppler spread, respectively. In many existing methods the fading caused by vehicular channel is been investigated and its major cause over MAI is expressed analytically. Though the Performance results obtained using Pilot aided channel estimation (CE) approach Mitigate these problems but it comes with maximum complexity and complex computations and has many limitations during real time hardware implementation. Moreover, all these estimation methods are robust for multiuser systems like MCCDMA with combined multiuser detection over MIMO where the composite signals are receives from all the users. However, it is also proved that all multi carrier systems have the limitations on its sensitivity to peak-to-average power ratio and its performance degradation over vehicular networks due to its time varying channel effects. Interference caused by time delay during multipath propagation can be solved by trading the spectral efficiency.
High spectral efficiency and its robustness to random time varying behavior of the wireless channels in multi-users environment multi carrier code division multiple access (MCCDMA) has been emerged as an ideal wireless transmission technique for next generation high speed wireless systems [1]. In most cases the research on the performance analysis of multi rate multicarrier code-division multiple-access (MCCDMA), composing of MIMO single-carrier systems in frequency-selective Rayleigh fading [2], a frequency domain spreading gain and equalization in CDMA system [3], Linear MMSE user detection techniques for better error rate performance [4], the channel capacity extension through diversity combining and space-time block coded multi carrier CDMA systems with moderate spreading codes over frequency selective Rayleigh fading channels [5] etc. Existing solutions for addressing the MAI problems over longer delay channel using joint estimation [6] is not adequate. Performance results obtained using maximum likelihood (ML) approach mitigates these problems [7] but it comes with maximum complexity and complex computations and has many limitations during real time hardware implementation. Moreover, all these estimation methods are robust for multiuser systems like MCCDMA with combined multiuser detection over MIMO where the composite signals are receives from all the users.

\section{Related Works}

Pilot-information based channel estimation is carried in CDMA system [8] for mitigating multipath channel using MMSE detector. In all existing methods, pilot-aided channel MMSE estimator is widely used for MIMO DSCDMA communication systems due to its superior performance over Rayleigh fading channel as well as 
additive noise channels. And moreover all types of multiple access interference (MAI) are modeled as additive noise [9]. Here in this work longer delay channel parameters is developed with maximum delay spread for noise interferences and Gaussian approximation is carried out for incorporating MAI. By exploiting unified Doppler effects one can extend it to evaluate under the fast fading channels to any other multipath fading channel. In this paper simulation of longer delay selective fading mobile radio channel environment is designed for MIMO multicarrier CDMA systems. Secondly, the performance of unique correlative variable length spread-spectrum code and its robustness to the multiple access interference (MAI) with variable rate transmission is analyzed. Thirdly, the analysis is applied to higher end constellations (QAM) MIMO systems with enhanced MMSE equalizer and its superiority is compared with ML approach.

\section{Proposed Method using MCCDMA System Model}

The generic performance analysis of our proposed MCCDMA system includes both the MIMO and variable length spreading code for multi user transmission schemes that are exploiting diversity gain from multi transmission and capable of providing significant error reduction using linear MMSE estimator which is not adequate for mitigating the MAI in any multicarrier modulation techniques. The analysis also takes includes the interferences caused by the time varying multipath components which leads inter symbol interferences combined with generic MAI even with the presence of guard interval, which is commonly included in any multicarrier models by assuming maximum channel delay spread.

\subsection{System model}

In this paper, a multi rate MCCDMA transmitter model is designed using STBC MIMO with $\mathrm{N}$ transmit and $\mathrm{M}$ receive antennas as shown in Fig $1 \&$ Fig 2. Here we consider a frequency selective fading channel whose channel impulse response between the $\mathrm{X}^{\text {th }}$ symbol transmitted and $\mathrm{Y}^{\text {th }}$ symbol received is modeled as

$\mathrm{H}_{X Y}^{\mathrm{t}}(\mathrm{t})=\mathrm{h}_{\mathrm{XY}}^{\mathrm{t}}(\mathrm{MxN}) \cdot \mathrm{e}^{\mathrm{j} \phi \mathrm{t}} \delta(\mathrm{t})$

The symbol conversion module consists of higher end non binary signaling pass band modulation (16-QAM, 64-QAM) with frame length 8 times as that of delay insertion carried for ISI interference and it offers significant simplicity at the receiver side. And then unique correlative spreading codes are generated as per the weighted matrix according to the specifications has given in previous section. Besides the orthoganality metrics considered between two successive codes generated channel allocations are carried out according to the throughput and Qos level required for that particular mode of transmission and diversity gain is optimized accordingly. The ultimate goal is to prove the robustness of system model to the multiple access interference problems over longer delay channels within the optimal range of the Doppler constrains. At the receiver side, multi user data detection is carried with simplified linear MMSE estimator followed by maximal ratio combining for MIMO detection. The interference cancellation with orthogonal subcarriers is achieved through single tap using iterative successive interferences cancellation to provide tradeoff between design complexities over QoS requirements.

\subsection{Correlation Property}

The general architecture of a CC based MIMO-MCCDMA system is shown in Fig. 1. Both transmit spatial diversity and orthoganality through unique correlative spreading codes are supported at the same time and it also has a unique nature of variable length where it can switch between various levels of subcarrier modes during Multiplexing.

The random data generated for each user $b(k)_{1}^{N}$ is mapped using higher order QAM modulation and corresponding correlative codes are generated based on sequence length, which performs correlation for mitigating multiple access interferences. Finally after the inclusion of cyclic extension space time matrix are incorporate for adding diversity which is necessarily implemented jointly over all the transmission branches and generated MIMO signals are up-converted to radio frequency and transmitted. It is worth noting that the channel STBC matrix is being consistent at the transmitter. Then, receiver module will perform according to the Diversity/spreading Controller used in the transmitter, where received signals are combined to attain diversity gain.

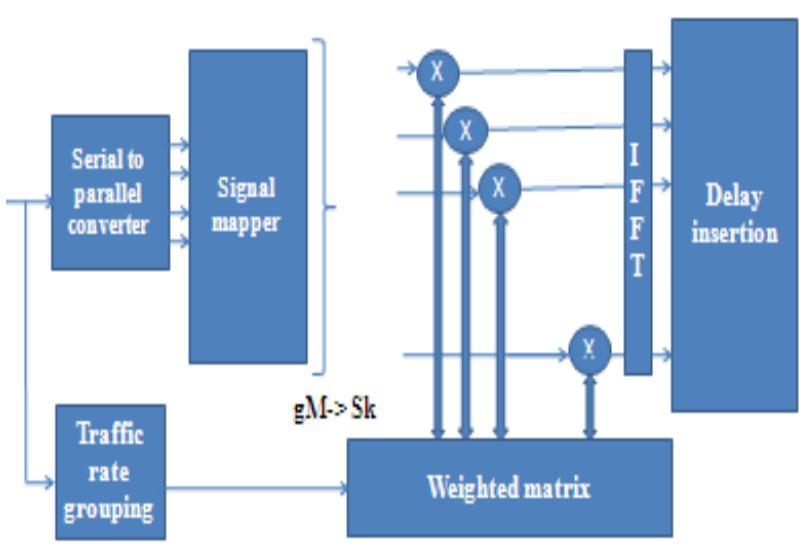

Fig. 1: Transmitter Architecture

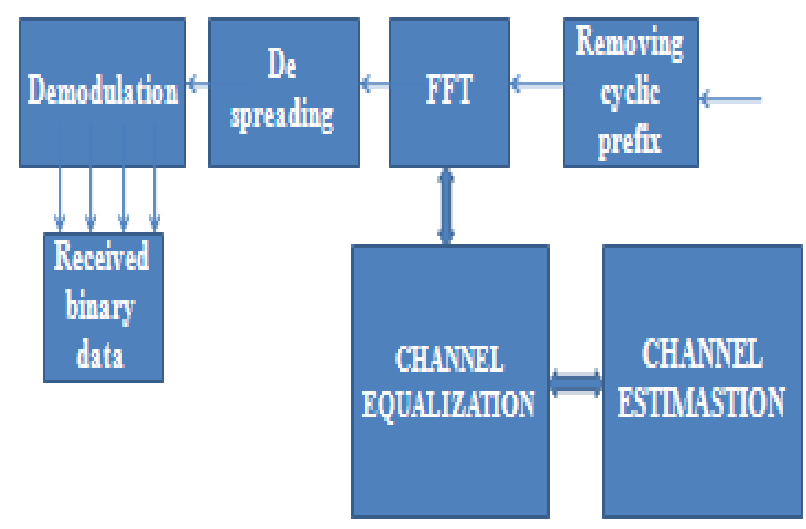

Fig. 2: Receiver Architecture

\subsection{MIMO MCCDMA System and Channel Model}

Conventional MIMO STBC schemes required complex state space matrix to fully exploit diversity to the higher end constellation. Due to high complexity the number of antennas used at the receiver side is optimized based on downlink side cellular system. Thus, there is a trade-off between the number of antennas and the constellation signal used. Apparently, any number of antennas can be used with any constellation signal. MIMO pre-coding technique for downlink system, which requires fully exploited channel state information (CSI) at the transmitter, has to be developed to solve the basic problems through channel state updation from the receiver side and it is assumed to be perfectly synchronized with transmitter to get best possible performance metrics of spreading gain from MC-CDMA system. Large-scale selective fading is been log-normally distributed and its deviations are independent of propagation environment and travelling distance as shown in Figure 3. 


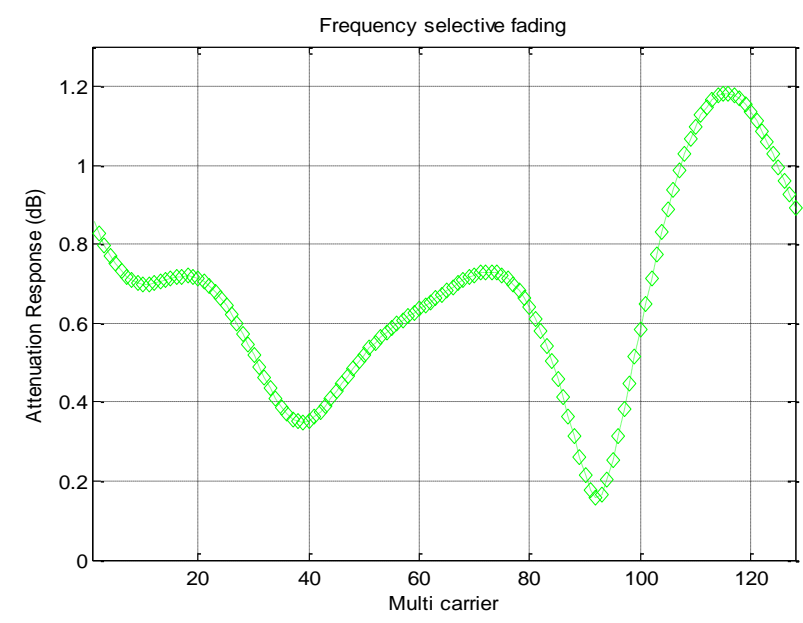

Fig. 3: Frequency selective fading channel response

To analyze the system performance over longer delay spread each path is allowed to experience certain degree of symbol interferences within a group. Form in ITU channel specifications Channel B, corresponds to high delay spread with highest possible average rms delay spread as per channel model specifications. Here Channel B channel matrix is used to analyze the system performance with worst case timing offset.

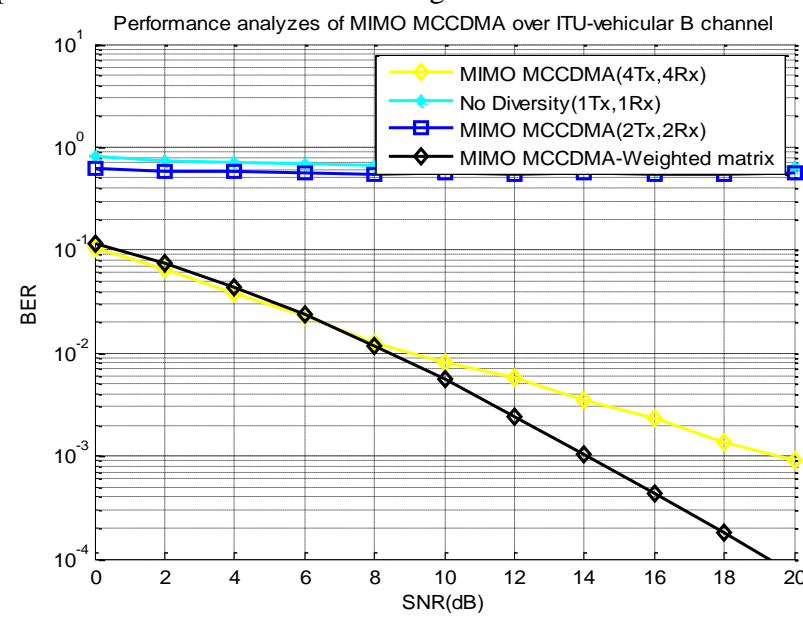

Fig. 4: BER analyzes of MIMO MCCDMA using 64-QAM

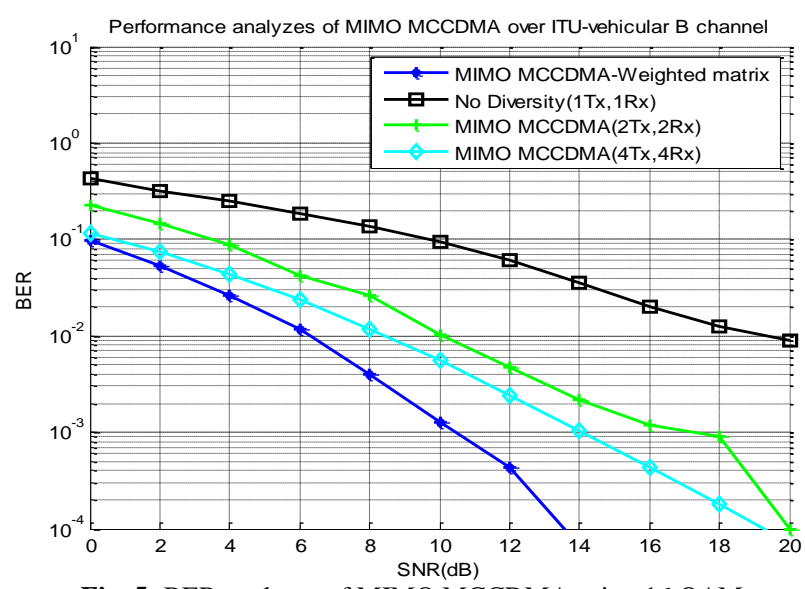

Fig. 5: BER analyzes of MIMO MCCDMA using 16 QAM

Here we investigate the performance of the proposed MIMOMCCDMA with simulation parameters as summarized in table 1 . The system bandwidth is divided into 128 subcarriers and the complementary codes are used for spreading the modulated sequence based on channel allocation and QoS respectively. Figure 4 and Figure5 illustrates the BER performance of different
MIMO diversity schemes under the time-varying with 16-QAM and 64-QAM. The proposed weighted matrix driven model outperforms conventional Gold sequence method and its performance is evaluated for comparison.

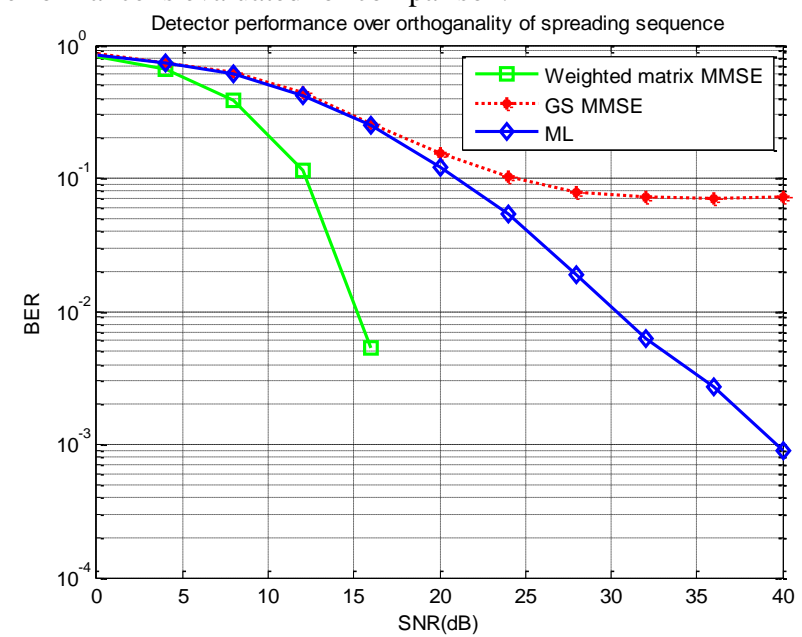

Fig. 6: Signal detector performance analyzes over Multipath Channel Environment

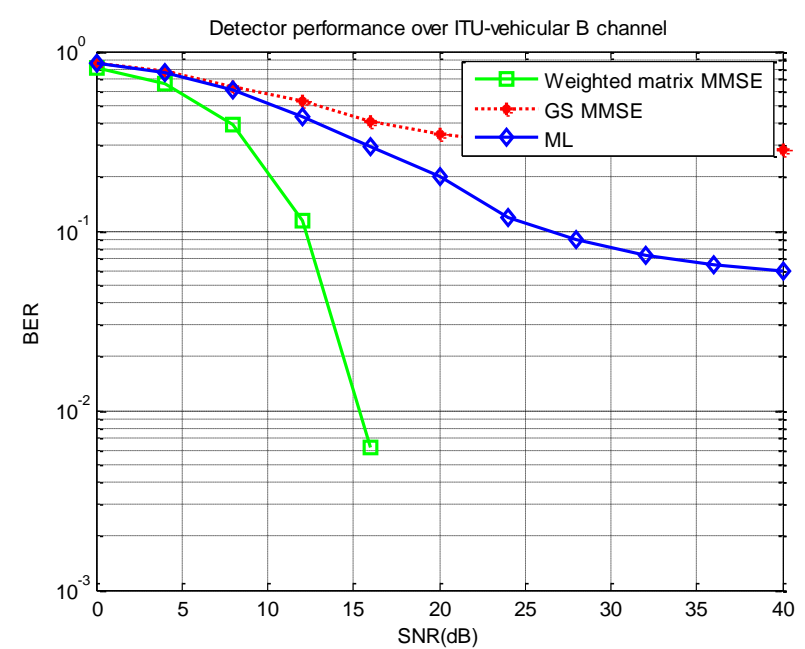

Fig. 7: Signal detector performance analyzes over ITU-B channel environment

\section{Performance Results}

\subsection{Simulation Results and Anayzes}

In this section, we analyze the impact of complementary codes used for spreading gain over time varying channel in MIMO systems. The channel model consist both the time delay for multipath longer delay and the Doppler frequency shift driven by high mobility channel model for finite trade off comparison between weighted matrix driven MCCDMA system over Gold sequence model. It is noted that, the construction of variable rate codes incorporate orthogonal matrices in MCCDMA system and satisfying the interference-free MIMO communication. Another commonly used set of empirical channel models is that specified in ITU-R recommendation M.1225. The recommendation specifies three different test environments: Indoor office, outdoorto- indoor pedestrian, and vehicular - high antenna. Since the delay spread can vary significantly, the recommendation specifies two different delay spreads for each test environment: low delay spread (A), and medium delay spread (B) are shown in figure 7 \& figure 8 


\subsection{Simulation Parameters}

Table 1: Percentage Occurrence and associated RMS Delay Spread for ITU Channel

\begin{tabular}{|c|c|r|c|r|}
\hline \multirow{2}{*}{ Test environment } & \multicolumn{2}{|c|}{ Channel A } & \multicolumn{2}{c|}{ Channel B } \\
\cline { 2 - 5 } & $\begin{array}{c}\text { r.m.s. } \\
(\mathrm{ns})\end{array}$ & $\begin{array}{c}P \\
(\%)\end{array}$ & $\begin{array}{c}\text { r.m.s. } \\
(\mathrm{ns})\end{array}$ & $\begin{array}{c}P \\
(\%)\end{array}$ \\
\hline Indoor office & 35 & 50 & 100 & 45 \\
\hline $\begin{array}{c}\text { Outdoor to indoor and } \\
\text { pedestrian }\end{array}$ & 45 & 40 & 750 & 55 \\
\hline
\end{tabular}

Table 2: Attributes Considered for the Experimental Setup

\begin{tabular}{|c|c|}
\hline FFT Size & 128 \\
\hline Spreading code used & Gold Sequence and Complementary codes \\
\hline Modulation type used & 16-QAM,64-QAM \\
\hline Channel Model & Rayleigh and ITU Vehicular B Channel \\
\hline
\end{tabular}

Subsequently, the diversity encoded error performance of MMSE is inspected. It is assumed that linear spacing is given to each channel taps which is equal to the transmission block length for Rayleigh channel model. ML based approach is outperformed the conventional MCCDMA model with MMSE detector as expected. But proposed weighted matrix MMSE model shows significant performance improvement as compared to ML as shown in Figure 6. One major drawback of MMSE equalization comes from larger constellation sizes such that the error rate increases excessively with higher order mapping sizes is mitigated with proposed model as shown in Figure 5, where 64-QAM mapping is used for error probability evaluation.

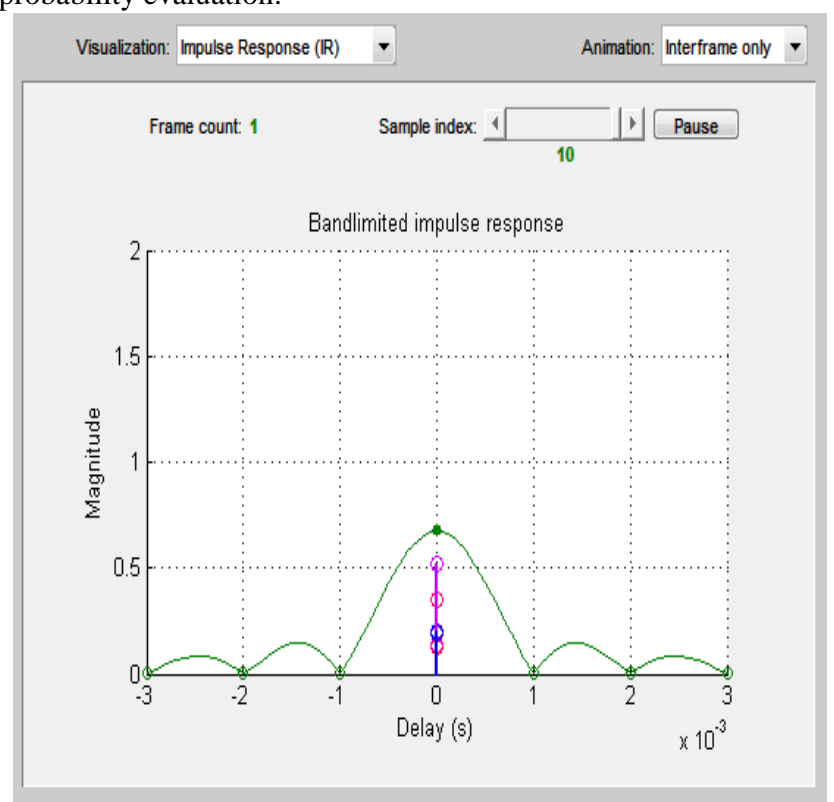

Fig. 8: ITU-Vehicular B longer delay spread channel response

The performance of proposed MCCDMA model is evaluated in ITU vehicular channel environment according to the ITU-R M.1225 specifications. Here, channel B, corresponds to high mobility channel with moderate multipath delay profile as shown in Figure 6. On the other hand predefined Doppler frequency is included to incorporate wide ranges of subcarrier interferences.

\section{Conclusion}

In this work, we carried out performance metrics of weighted matrix driven spreading scheme over multiple antennas using linear MMSE detector. First, we derive analytical expressions of the elementary longer delay spread channel with different multipath and channel delay bound assumptions. Then, we apply the obtained expressions to prove its achievable error rate performance and compare them with state-of-the-art ML scheme.
It is proved that the error rate measure of MIMO-MCCDMA systems mainly depends on the constellation points design complexity. The proposed weighted matrix MCCDMA system can able to achieve better BER reduction with consistent performance retention. In simulation results we proved that the linear MMSE achieves better performance with lower complexity than all other state-of-the-art-results.

\section{References}

[1] H. Atarashi, N. Maeda, S. Abeta, and M. Swahashi, "Broadband packet wireless access based on VSF-OFCDM and MC/DSCDMA," in Proc. of IEEE PIMRC'02, Lisbon, Portugal, Sept. 2002, pp. 992-997.

[2] Xu H, Chizhik D, Huang H \& Valenzuela R (2004) A generalized space-time multiple-input multiple-output (MIMO) channel model. IEEE Transactions on Wireless Communications 3(3): 966-975.

[3] Gui X \& Ng TS (1999) Performance of asynchronous orthogonal multicarrier CDMA system in frequency selective fading channel. IEEE Transactions on Communications 47(7): 1084-1091.

[4] Hélard JF, Baudais JY \& Citerne J (2000) Linear MMSE detection techniques for MC-CDMA. Electronics Letters 36(7): 665-666.

[5] $\mathrm{Hu} \mathrm{X} \mathrm{\&} \mathrm{Chew} \mathrm{YH} \mathrm{(2004)} \mathrm{On} \mathrm{the} \mathrm{capacity} \mathrm{of} \mathrm{multi} \mathrm{code} \mathrm{and}$ variable spreading gain multirate space-time block coded multicarrier CDMA systems over frequency selective Rayleigh fading channels. Proc. IEEE Vehicular Technology Conference, Los Angeles, USA, 1: 353-357.

[6] E. P. Simon, L. Ros, H. Hijazi, and M. Ghogho, "Joint carrier frequency offset and channel estimation for OFDM systems via EM algorithm in the presence of very high mobility," IEEE Trans. Signal Process., vol. 60, no. 2, pp. 754-765, Feb. 2012.

[7] Muneer P. and Sameer S.M., "Pilot aided joint estimation of doubly selective channel and carrier frequency offset in high mobility OFDMA uplink," Proc. 19th National Conference on Commun. (NCC 2013), IITDelhi, pp. 1-5, Feb. 2013.

[8] M. N. Iqbal and M. Moinuddin, "Pilot-aided rayleigh fading channel estimation using mmse estimator for ds-cdma system," in Multitopic Conference (INMIC), 2011 IEEE 14th International, pp. 347-350, IEEE, 2011

[9] R. K. Morrow Jr and J. S. Lehnert, "Bit-to-bit error dependence in slotted ds/ssma packet systems with random signature sequences," Communications, IEEE Transactions on, vol. 37, pp. 1052-1061, Oct 1989. 